\title{
Testicular cancer in young men: the search for causes of the epidemic increase in the United States
}

\author{
LINDA MORRIS BROWN, LINDA M POTTERN, AND ROBERT N HOOVER \\ From the Epidemiology and Biostatics Program, National Cancer Institute, National Institutes of Health, \\ Bethesda, MD 20892, USA
}

SUMMARY A case-control study of 271 men with testicular cancer and 259 controls was conducted in the Washington, DC area to evaluate whether suggested risk factors could be responsible for the epidemic increases in testicular cancer in young men. No substantial risks were associated with a history of groin hernia operation, the common childhood diseases, allergies, $x$ rays below the waist, venereal disease, vasectomy, or external means of elevating the temperature of the testis. Excess risks were associated with a history of undescended testis $(R R=3 \cdot 7, C I=1 \cdot 5-9 \cdot 5)$, testicular trauma $(\mathrm{RR}=2 \cdot 6, \mathrm{CI}=1 \cdot 6-4 \cdot 2)$, and mumps orchitis $(\mathrm{RR}=5 \cdot 8, \mathrm{CI}=0 \cdot 7-129 \cdot 7)$. It is unlikely, however, that any of these conditions has increased sufficiently over time to markedly affect the testicular cancer incidence patterns. Therefore, while the risk factors identified in this paper are of epidemiological interest, they do not account for the increase in testicular cancer in young men.

Over the past several decades there have been dramatic increases in the incidence of testicular cancer, the most striking increases occurring among young men aged 15-44. ${ }^{1}$ An aetiological role for undescended testis, groin hernia, testicular trauma, mumps, mumps orchitis, elevated testicular temperature, and hormonal, prenatal, and occupational factors in the development of young adult testicular cancers has been suggested by a number of studies. ${ }^{2-12}$ However, previous researchers have in general been hampered by small study populations, which made it difficult to test those hypotheses. Therefore, we conducted a case-control study of testicular cancer in young men at three large referral centres to test the hypotheses generated by previous studies and to evaluate whether any of the suggested risk factors could be responsible for the epidemic increases in this cancer.

\section{Methods}

All testicular cancer cases aged 18-42 who had been newly diagnosed between 1 January 1976 and 30 June 1981 and referred to one of three collaborating medical centres in the Washington, DC area: National Institutes of Health Clinical Center (NIHCC), Uniformed Services University, Naval Hospital (USUNH), and Uniformed Services University, Walter Reed Army Medical Center (WRAMC) were selected for study. Cases diagnosed prior to 1979 were ascertained from tumour registry, hospital admissions, urology, and pathology records at the three centres and were interviewed over the telephone. Face-to-face interviews were conducted in the hospital for those cases seen on the oncology, surgical, and urology wards during 1979-81. Twenty-seven deceased testicular cancer patients diagnosed prior to 1979 were excluded from the study.

Controls were patients at the same hospital as the cases diagnosed with a malignancy other than cancer of the genital tract. They were similar to the cases in age ( \pm 2 years), race, vital status, and year of diagnosis. Controls diagnosed during 1976-78 were identified from computerised discharge logs at NIHCC and WRAMC and from tumour registry records at USUNH and were interviewed over the telephone. Controls diagnosed during 1979-81 were identified on the oncology, surgical and medical wards and were interviewed in the hospital in the same manner as the cases. The medical records of all patients were reviewed to confirm the diagnosis.

Interviews of the study subjects were conducted during 1979-81. A standardised questionnaire was used by trained interviewers to obtain detailed information on the study subject's medical history (eg, childhood diseases, hernia, undescended testis, trauma to the testis, venereal disease, and diagnostic $x$ rays), personal history (eg, type of underwear and bathing habits), smoking history, and demographic information. Due to the young age of the study subjects, the measure of residence used in the analysis 
was usual residence as a child. Subjects were asked to classify this area as being either rural (farm country or small town/community) or urban (city or suburb).

Telephone interviews were conducted with the study subjects' mothers to elicit information on the sons' in-utero exposures and childhood medical history. ${ }^{2}$ Information was provided by the mothers on undescended testis, inguinal hernia, mumps orchitis, and childhood diseases. Information from the sons' questionnaires were used to supplement the mothers' responses when they were either unknown or unavailable.

The measure of association between testicular cancer and variables of interest was the relative risk (RR), approximated by the odds ratio. ${ }^{13}$ Ninety-five per cent confidence intervals $(\mathrm{CI})$ were calculated according to the method described by Gart. ${ }^{14}$ Summary RR estimates adjusted for the stratifying (matching) variables hospital and age at diagnosis were calculated by the Mantel-Haenszel method. ${ }^{15}$ Since the RR adjusted for the matching variables were similar in magnitude and direction to the unadjusted $\mathbf{R}$, the crude $R \mathbf{R}$ are presented in this paper unless otherwise specified. Type of patient interview, in person or telephone, did not affect the risk estimates. Fisher's exact test was used to calculate a lower bound CI when the number of controls in a cell was zero. ${ }^{13}$ The population attributable risk associated with undescended testis was estimated assuming that prevalence of an undescended testis in the general male population is about $2 \cdot 5 \%$ by age 3 years. ${ }^{16-18}$ Chisquare tests of homogeneity were used to test for case-control differences in referral patterns. ${ }^{13}$ Mantel's extension test ${ }^{19}$ was used to test for trends (two-tailed) in risk related to cigarette consumption.

\section{Results}

Completed valid interviews were obtained for 271 cases $(88.0 \%)$ and 259 controls $(89 \cdot 9 \%)$. Four per cent of the cases and $2 \%$ of the controls refused to be interviewed. The remainder were either unlocatable or unavailable for interview. One control with unreliable information was dropped from the analysis. Sixty-nine per cent of the cases and $71 \%$ of the controls were interviewed in person in the hospital, while $31 \%$ of the cases and $29 \%$ of the controls were interviewed at home over the telephone. The malignancies among the controls were Hodgkin's disease $(29 \%)$, nonHodgkin's lymphoma (18\%), melanoma (17\%), soft tissue sarcoma $(7 \%)$, bone tumour $(7 \%)$, leukaemia $(7 \%)$, and other $(15 \%)$. The histological types of testicular cancer were embryonal carcinoma $(25 \%)$, teratocarcinoma $(25 \%)$, seminoma $(22 \%)$, mixed tumours with seminoma $(14 \%)$, mixed tumours without seminoma $(7 \%)$, teratoma $(5 \%)$, and other or unknown (2\%). The percentage of study subjects seen at each hospital was: NIHCC, $48 \%$; USUNH, $22 \%$; and WRAMC, $30 \%$. To assess the possibility of referral bias, a chi square for homogeneity with regard to the geographic distribution (ie, Maryland, DC or Virginia; south; northeast; midwest; west; and foreign country) of the referring physicians of cases and controls was conducted. There were no significant differences in their referral patterns $\left(\chi^{2}=7 \cdot 4 ; p=0 \cdot 19\right)$.

The study population was $98 \%$ white with $70 \%$ between the ages of 16 and 30 years. The mean age at diagnosis was the same for cases and controls, 270 years. The percentage of study subjects with more than a high school education was similar for cases $(51 \%)$ and controls $(52 \%)$. Mean height obtained from the

Table 1 Relative risks of testicular cancer according to demographic characteristics

\begin{tabular}{|c|c|c|c|c|}
\hline Risk factor & $\begin{array}{l}\text { No. of } \\
\text { cases }\end{array}$ & $\begin{array}{l}\text { No. of } \\
\text { controls }\end{array}$ & $R R$ & $95 \% C I$ \\
\hline \multicolumn{5}{|l|}{ Childhood residence } \\
\hline Rural & 132 & 144 & $1 \cdot 0$ & -- \\
\hline Urban & 130 & 108 & $1 \cdot 3$ & $0.9-1.9$ \\
\hline Both & 9 & 7 & 1.4 & $0 \cdot 5-4.3$ \\
\hline \multicolumn{5}{|l|}{ Religion } \\
\hline Protestant & 167 & 173 & $1 \cdot 0$ & - \\
\hline Catholic & 85 & 68 & $1 \cdot 3$ & $0.9-1.9$ \\
\hline Jewish & 4 & 10 & 0.4 & $0 \cdot 1-1 \cdot 5$ \\
\hline Mormon & 5 & 0 & & $0.9-\infty$ \\
\hline Other & 10 & 8 & 1.29 & $0.5-3.7$ \\
\hline \multicolumn{5}{|l|}{ Marital status } \\
\hline Ever married & 141 & 135 & 1.0 & - \\
\hline Never married & 130 & 124 & 1.0 & $0.7-1.4$ \\
\hline \multicolumn{5}{|l|}{ Infertility* } \\
\hline No & 127 & 121 & $1 \cdot 0$ & 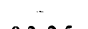 \\
\hline Yes (saw doctor) & 8 & 9 & 0.8 & $0 \cdot 3-2 \cdot 5$ \\
\hline Yes (no doctor) & 5 & 5 & 1.0 & $0.2-3.9$ \\
\hline
\end{tabular}

- Includes only married study subjects. 
medical records was 70.0 inches for cases and 69.5 inches for controls.

Table 1 presents the RR of testicular cancer for selected demographic characteristics. Although none of the RRs was statistically significant, there was an increased risk for usual childhood residence in a primarily urban rather than a primarily rural area. Compared to Protestant, a reduced risk was seen for Jewish and slightly elevated risks were seen for Catholic and no religion. Five cases and no controls reported being Mormon. No excess risk was seen according to marital status or infertility. The average age at marriage was 22 years for both cases and controls.

In order to evaluate the risks associated with two external means which have been speculated to elevate the temperature of the testis, study subjects were asked what type of underwear they usually wore and whether they usually took a shower or a bath when they bathed. There were no excess risks associated with wearing jockey type shorts versus boxer type shorts or with taking a bath instead of a shower (table 2).

RRs for testicular cancer according to history of specific medical conditions are presented in table 3.
Undescended testis was the only medical condition that was associated with a significantly elevated risk of testicular cancer: $\mathbf{R R}=3 \cdot 7$, yielding a population attributable risk estimate of $6.3 \%$. The risks were similar for subjects who were interviewed in person $(R R=3.3, C I=1 \cdot 1-10 \cdot 6)$ and over the telephone $(R R=4 \cdot 5, C I=0 \cdot 9-31 \cdot 3)$. The associations between testicular cancer risk and undescended testis and groin hernia have been explored in detail in another publication. ${ }^{3}$

No substantial differences were seen for histories of groin hernia operation uncomplicated by undescended testis, measles, chicken pox, allergies, $x$ rays below the waist, venereal disease (primarily gonorrhoea), and vasectomy. Specific allergies (eg, hayfever and trees/grasses) also were not related to testicular cancer risk. A decreased risk was seen for a history of receiving acne treatments from a doctor. A history of mumps orchitis was associated with an almost six-fold risk (based on the combined positive responses from two cases and six case mothers and one control and no control mothers), whereas the RR for a history of uncomplicated mumps was nonsignificantly reduced.

Table 2 Relative risks of testicular cancer according to external means of elevating temperature of the testis

\begin{tabular}{|c|c|c|c|c|}
\hline External means & $\begin{array}{l}\text { No. of } \\
\text { cases }\end{array}$ & $\begin{array}{l}\text { No. of } \\
\text { controls }\end{array}$ & $R R$ & $95 \% C I$ \\
\hline \multicolumn{5}{|c|}{ Usual type of underwear* } \\
\hline Boxer shorts & 36 & 25 & 1.0 & - \\
\hline Jockey shorts & 219 & 222 & 0.7 & $0.4-1 \cdot 2$ \\
\hline None & 15 & 11 & 0.9 & $0.3-2.7$ \\
\hline \multicolumn{5}{|c|}{ Usual bathing habits } \\
\hline Shower & 237 & 223 & 1.0 & - \\
\hline Bath & 31 & 32 & 0.9 & $0.5-1.6$ \\
\hline Both & 3 & 4 & 0.7 & $0.1-3.8$ \\
\hline
\end{tabular}

* excludes 1 case and 1 control who did not report a usual type of underwear.

Table 3 Relative risks of testicular cancer according to medical history

\begin{tabular}{|c|c|c|c|c|c|c|}
\hline \multirow{2}{*}{ Risk factor* } & \multicolumn{2}{|c|}{$\begin{array}{l}\text { No. of } \\
\text { cases }\end{array}$} & \multicolumn{2}{|c|}{$\begin{array}{l}\text { No. of } \\
\text { controls. }\end{array}$} & \multirow{2}{*}{$R R$} & \multirow{2}{*}{$95 \% C I$} \\
\hline & Yes & No & Yes & No & & \\
\hline Undescended testis & 25 & 246 & 7 & 252 & $3 \cdot 7$ & $1.5-9.5$ \\
\hline Hernia operationt & 18 & 228 & 15 & 237 & $1 \cdot 2$ & $0.6-2.7$ \\
\hline Measles & 251 & 18 & 236 & 18 & $1 \cdot 1$ & $0.5-2.2$ \\
\hline Chicken pox & 246 & 18 & 236 & 19 & 1.1 & $0.5-$ \\
\hline Mumps & 200 & 66 & 206 & 49 & 0.7 & $0.5 \quad 1 \cdot 1$ \\
\hline Mumps orchitis & 6 & 265 & 1 & 258 & $5 \cdot 8$ & $0.7-129 \cdot 7$ \\
\hline Treated for acne & 24 & 247 & 33 & 226 & 0.7 & $\begin{array}{ll}0.4 & 1.2\end{array}$ \\
\hline Allergy & 75 & 196 & 75 & 184 & 0.9 & 0.6 \\
\hline$x$ rays below waist & 47 & 224 & 45 & 214 & 1.0 & $0.6-1.6$ \\
\hline Venereal disease & 22 & 249 & 22 & 237 & $1 \cdot 0$ & $0.5-1.8$ \\
\hline Vasectomy & 5 & 266 & 5 & 254 & 1.0 & $0.3-3.3$ \\
\hline
\end{tabular}

* Don't know responses are excluded from table.

t Excludes subjects with concommitant undescended testis and hernia. 
A history of smoking at least 100 cigarettes during their lifetime was reported by $62.4 \%$ of the cases and by $53.3 \%$ of the controls $(R R=1 \cdot 5, C I=1 \cdot 0-2 \cdot 1)$. The RR for smoking was significantly elevated at NIHCC $(\mathrm{RR}=2 \cdot 1, \quad \mathrm{CI}=1 \cdot 2-3 \cdot 6)$ but not at USUNH $(R R=1 \cdot 2, \quad C I=0 \cdot 5-2 \cdot 6)$ or WRAMC $(R R=1 \cdot 0$, $\mathrm{CI}=0 \cdot 5-2 \cdot 1)$. Since the RRs for the number of packs of cigarettes smoked per day were similar for USUNH and WRAMC (military hospitals), their combined results are presented in table 4 . There was a significant trend with number of packs of cigarettes smoked at NIHCC ( $p$ for trend $=0.022$ ), although the RR declined for the highest smoking category. No doseresponse was seen at the two military hospitals. A similar pattern of risk was seen at NIHCC for the number of years smoked (ie, a drop in the RR for subjects smoking the greatest number of years). Results remained similar when controls with leukaemia and other cancers possibly related to smoking were removed.

The RR for study subjects reporting a history of trauma to the testis was significantly elevated, $\mathrm{RR}=2 \cdot 6(\mathrm{CI}=1 \cdot 6-4 \cdot 2 ; 79$ cases, 35 controls $)$ and remained elevated, $R R=2 \cdot 3(\mathrm{CI}=1 \cdot 3-4 \cdot 1 ; 45$ cases, 21 controls) when those reporting a trauma less than ten years before their cancer diagnosis were removed from the analysis. For this latter group, the average ages for first trauma to the testis were 11.6 years for the cases and 11.1 years for the controls. For both cases and controls, mean age at last trauma was 12.7 years. There were no differences in risk for the most frequently reported sources of trauma, being kicked $(R R=2 \cdot 0, C I=1 \cdot 3-3 \cdot 9)$, injured in an accident or fall $(R R=2 \cdot 3, C I=0 \cdot 5-11 \cdot 2)$, and injured on a bicycle $(\mathrm{RR}=1 \cdot 9, \mathrm{CI}=0 \cdot 5-7 \cdot 8)$.

\section{Discussion}

This case-control study was designed to investigate a number of factors potentially related to the risk of testicular cancer in young men and to evaluate whether any of these factors could be responsible for the dramatic increases in incidence over time.

A variety of sociodemographic characteristics were examined but none was significantly elevated. A slight excess risk was found for spending one's childhood in an urban rather than a rural area. This is consistent with a study reported in Denmark ${ }^{20}$ but contrary to studies in England and Wales, ${ }^{21}$ the Netherlands, ${ }^{22}$ and Upstate New York. ${ }^{23}$ No marked differences were seen according to religion in which the study subjects were raised, with the exception of the Mormon religion. There was an apparent excess of Mormons with testicular cancer (although based on small numbers). However, Lyon et $a l^{24}$ reported no difference in the age-adjusted rates of testicular cancer in Mormons and Non-Mormons in Utah.

It has been suggested that external factors that elevate the temperature of the testis have been associated with increased risk of testicular cancer. ${ }^{6-9}$ However, in this study there were no differences between cases and controls in the type of underwear usually worn or their typical bathing habits.

It has been well documented in the literature that individuals with undescended testis are at an increased risk for developing testicular cancer. In our casecontrol study, $10 \%$ of the cases and $3 \%$ of the controls reported a history of undescended testis, resulting in a significantly elevated risk of $3 \cdot 7$. This is consistent with the findings of other case-control studies which have reported risks ranging from $2 \cdot 5$ to $17 \cdot 1 .^{3-8} 11$

Inconsistent findings regarding the degree and significance of the risk of testicular cancer associated with groin hernia have been reported. ${ }^{3-57811}$ In our study, those men who had a history of a hernia operation but no history of undescended testis did not have a significant excess risk of testicular cancer. The reduced risk seen for acne treatments by a doctor was not significant. However, the reduction in risk was similar to that reported by Depue et al. ${ }^{4}$

Table 4 Relative risks of testicular cancer according to number of packs of cigarettes smoked and type of hospital

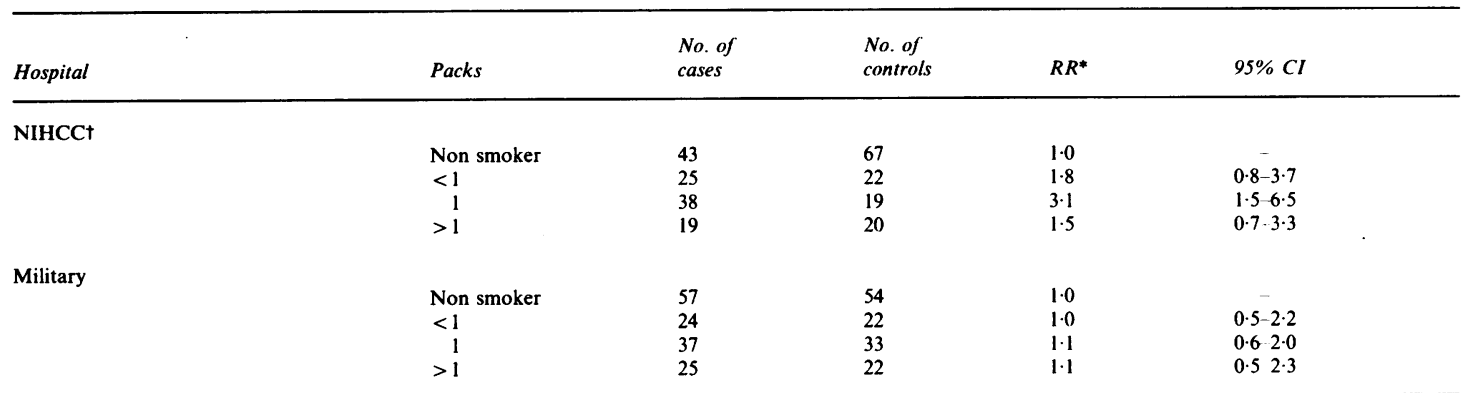

\footnotetext{
* Risks relative to 1.0 for non-smokers at each hospital.
}

† Excludes 2 cases with unknown smoking habits. 
In agreement with other studies 5825 we found no significant excess risks associated with a history of measles, chicken pox, uncomplicated mumps, or venereal disease. Several clinical reports ${ }^{12}$ and a population-based study of mumps orchitis ${ }^{10}$ have suggested a relation between mumps orchitis and testicular cancer. In our study, mumps orchitis was reported for six cases and one control-a positive, although nonsignificant, association. However, the structure of this question, asking those subjects who reported a history of mumps whether it involved the testis, may have encouraged recall bias. Also, it is not clear whether study subjects could discriminate between mumps orchitis and other testicular conditions such as epididymitis. ${ }^{26}$

The relation between cigarette smoking and testicular cancer has not been well studied. In our study, there was a significantly elevated risk of 1.5 for ever smokers of cigarettes using the total study group. However, when cigarette smoking was stratified by hospital, the excess risk was restricted to those cases referred to NIHCC. Therefore, our results in conjunction with the previous negative findings reported in the literature ${ }^{5,8}$ suggest that a causal relation between cigarette smoking and testicular cancer is unlikely.

To investigate the association between testicular trauma and testicular cancer, study subjects were asked if they had ever had any trauma or injury to the testis and the specific type of trauma. Since trauma to the testis may have caused some study subjects to seek medical care, thus bringing otherwise asymptomatic testicular tumours to the attention of a physician, only those traumas that occurred at least one year prior to diagnosis were included in the analysis. A risk of 2.6 was associated with having a trauma to the testis. Risks were elevated for injuries incurred during the following situations: riding a bicycle, playing sports, or having an accident or fall. Risks remained elevated when study subjects whose first trauma occurred less than ten years prior to diagnosis were excluded. Only one previous case-control study has addressed the issue of testicular cancer following trauma or injury to the testis. ${ }^{5}$ That study asked specific questions on participation in sports activities and found excess risks with bicycling and horseback riding. Although testicular trauma appears to be related to the risk of testicular cancer, the positive association should be viewed cautiously because of the problem of recall bias.

As in other investigations, the associations found or the failure to find associations could be due to bias. Selection bias does not appear to be a factor in this study since response rates were high and the controls chosen had referral patterns similar to those of the cases. However, it is possible that some of the childhood exposures studied could play an aetiological role in other cancers in young adults. If this is the case, it is possible that an important association of interest could have been 'matched out' since the comparison group was composed of young men with other cancers. Nevertheless, the choice of other cancers for the comparison population was purposeful since childhood exposures may be subject to recall bias. Because cases and controls both developed a malignancy, these types of exposures should not be remembered differently by cases and controls. It is possible, however, that testicular cancer cases may have been more likely than the controls to recall a history of other urogenital conditions or injuries because of the location of the tumour.

This study did not find substantial risks associated with external means which have been speculated to elevate the temperature of the testis or with a history of groin hernia operation, the common childhood diseases, allergies, $x$ rays below the waist, venereal disease, vasectomy, or cigarette smoking. It did find excess risks due to undescended testis, mumps orchitis, and testicular trauma. However, none of these conditions has increased sufficiently over time to markedly affect the testicular cancer incidence patterns.

Reasons for the increases in testicular cancer incidence, especially among young men, remain elusive. The increases do not appear to be related to improved diagnostic practices or to risk factors identified in this and other case-control studies.

We thank Dr Nasser Javadpour, NIHCC, Dr Kevin J O'Connell, USUNH, and Dr Ray E Stutzman, WRAMC, for allowing us access to their patients; Ms Jeanne Rosenthal, Ms Kathy Weber, Ms Judith Walsh, Ms Sandra Becker, and the staff of Westat Inc for data management and interviewing; and Ms Roselyn Weil, NCI, for data collection and interviewing.

Address reprint requests to: Ms L M Brown, Room 3C15, Landow Building, National Cancer Institute, National Institutes of Health, Bethesda, MD 20892, USA.

\section{References}

${ }^{1}$ Brown LM, Pottern LM, Hoover RN, et al. Testicular cancer in the United States: Trends in incidence and mortality. Int J Epidemiolol 1986; 15: 164-70.

2 Brown LM, Pottern LM, Hoover RN. Prenatal and perinatal risk factors for testicular cancer. Cancer Res 1986; 46: 4812-6. 
${ }^{3}$ Pottern LM, Brown LM, Hoover RN, et al. Testicular cancer risk among young men: The role of cryptorchidism and inguinal hernia. $J N C I$ 1985; 74: 377-81.

${ }^{4}$ Depue RH, Pike MC, Henderson BE. Estrogen exposure during gestation and risk of cancer of the testis. JNCI 1983; 71: 1151-5.

5 Coldman AJ, Elwood JM, Gallagher RP. Sports activities and risk of testicular cancer. $B r J$ Cancer 1982; 46: 749-56.

${ }^{6}$ Loughlin JE, Robboy SJ, Morrison AS. Risk factors for cancer of the testis. $N$ Eng $J$ Med 1980; 303: 112.

${ }^{7}$ Schottenfeld D, Warshauer ME, Sherlock S, et al. The epidemiology of testicular cancer in young adults. $\mathrm{Am} \mathrm{J}$ Epidemiol 1980; 112: 232-46.

${ }^{8}$ Henderson BE, Benton B, Jing J, et al. Risk factors for cancer of the testis in young men. Int J Cancer 1979; 23: 598-602.

${ }^{9}$ Lin RS, Kessler II. Epidemiologic findings in testicular cancer. Abstract Am J Epidemiol 1979; 110: 357.

${ }^{10}$ Beard CM, Benson RC, Kelalis PP, et al. The incidence and outcome of mumps orchitis in Rochester, Minnesota, 1935 to 1974. Mayo Clinic Proceedings 1977; 52: 3-7.

11 Morrison AS. Cryptorchidism, hernia, and cancer of the testis. JNCI 1976; 56: 731-3.

12 Kaufman JJ, Bruce PT. Testicular atrophy following mumps. A cause of testis tumour? Br J Urol 1963; 35: 67-9.

${ }^{13}$ Fleiss JL. Statistical methods for rates and proportions. New York; Wiley, 1981: 24-26, 61-71, 160-165.

${ }^{14} \mathrm{Gart}$ JJ. The comparison of proportions: Review of significance tests, confidence intervals, and adjustments for stratification. Rev Int Stat Inst 1971; 39: 148-69.
${ }^{15}$ Mantel N, Haenszel W. Statistical aspects of the analysis of data from retrospective studies of disease. $J$ Natl Cancer Inst 1959; 22: 719-48.

${ }^{16}$ Mau G, Schnakenburg Kv. Maldescent of the testes-an epidemiological study. Europ J Pediat 1977; 126: 77-84.

${ }^{17}$ Depue RH. Maternal and gestational factors affecting the risk of cryptorchidism and inguinal hernia. Int $J$ Epidemiol 1984; 13: 311-8.

${ }^{18}$ Levin ML, Bertell R. Re: Simple estimation of population attributable risk from case-control studies. $\mathrm{Am} J$ Epidemiol 1978; 108: 78-9.

${ }^{19}$ Mantel N. Chi-square test with one degree of freedom, extensions of Mantel-Haenszel procedure. J Am Stat Assoc 1963; 58: 690-700.

${ }^{20}$ Clemensen J. Testis cancer incidence-suggestion of a world pattern. Int J Androl 1981; Suppl 4: 111-22.

${ }^{21}$ Lipworth L, Dayan AD. Rural preponderance of seminoma of the testis. Cancer 1969; 23: 1119-21.

22 Talerman A, Kaalen JGAH, Fokkens W. Rural preponderance of testicular neoplasms. Br J Cancer 1974; 29: 176-8.

${ }^{23}$ Graham S, Gibson R, West D, et al. Epidemiology of cancer of the testis in upstate New York. JNCI 1977; 58: 1255-61.

${ }^{24}$ Lyon JL, Gardner JW, West DW. Cancer incidence in Mormons and Non-Mormons in Utah during 1967-75. JNCI 1980; 65: 1055-61.

${ }^{25}$ Morrison AS. Some social and medical characteristics of army men with testicular cancer. Am J Epidemiol 1976; 104: 511-6.

${ }^{26}$ Ehrengut W, Schwartau M. Mumps orchitis and testicular tumours. Br Med J 1977; 2: 191. 